\title{
Early monitoring for detection of antituberculous drug-induced hepatotoxicity
}

\author{
Chang Min Lee, Sang Soo Lee, Jeong Mi Lee, Hyun Chin Cho, Wan Soo Kim, Hong Jun Kim, \\ Chang Yoon Ha, Hyun Jin Kim, Tae Hyo Kim, Woon Tae Jung, and Ok Jae Lee
}

Department of Internal Medicine, Gyeongsang National University Hospital, Jinju, Korea

Received: December 19, 2014

Revised : March 21, 2015

Accepted: May 11, 2015

\section{Correspondence to}

Sang Soo Lee, M.D.

Department of Internal Medicine, Gyeongsang National University Hospital, 79 Gangnam-ro, Jinju 52727 , Korea

Tel: +82-55-750-8932

Fax: +82-55-750-9496

E-mail:3939lee@naver.com
Background/Aims: We investigated the time of onset of antituberculous drug-induced hepatotoxicity (ADIH) and related characteristics.

Methods: Adult patients $(\mathrm{n}=1,031)$ treated with first-line antituberculous drugs between February 2009 and January 2013 were enrolled.

Results: Of the 1,031 patients, 108 patients (10.5\%) developed ADIH a mean of 39.6 \pm 43.7 days after treatment initiation. Twenty-eight patients $(25.9 \%)$ developed ADIH within 7 days, 73 (67.6\%) within 30 days, and the rest after 30 days. The $\leq$ 30-day group was characterized by higher peak alanine aminotransferase (ALT) level and a high proportion of patients with maintenance of first-line antituberculous drugs compared to the $>30$-day group. In subgroup analysis, the $\leq 7$-day group was characterized by higher baseline aspartate aminotransferase and ALT, high proportion of patients with maintenance of first-line antituberculous drugs, and high proportion of patients with extrapulmonary tuberculosis compared to patients with ADIH that developed beyond 7 days. In multivariate analysis, serum ALT > $40 \mathrm{IU} / \mathrm{L}$ (odds ratio [OR], 2.995; 95\% confidence interval [CI], 1.580 to 5.680; $p=0.001$ ) and presence of anti-hepatitis C virus (OR, 4.204; 95\% CI, 1.822 to 9.700, $p=0.001$ ) were independent risk factors for development of ADIH.

Conclusions: Approximately 70\% of the cases of ADIH occurred in the first month of antituberculous treatment, and were associated with continuation of the first-line drug regimen.

Keywords: Drug-induced liver injury; Drug monitoring; Tuberculosis; Republic of Korea

\section{INTRODUCTION}

Tuberculosis remains a global problem, with an estimated 8.6 million new cases reported in 2012 [1]. The standard treatment regimen of isoniazid, rifampicin, ethambutol, and pyrazinamide shortens the treatment period and increase treatment efficacy $[2,3]$. However, hepatotoxicity is common side effect of antituberculous treatment [4,5], and antituberculous drugs such as isoniazid, rifampicin and pyrazinamide are hepatotoxic [6].
Rates of antituberculous drug-induced hepatotoxicity (ADIH) ranging from $2.55 \%$ to $16.1 \%$ have been reported in several prospective studies [7-10]. Once hepatotoxicity occurs in a patient receiving antituberculous treatment, the treatment antituberculous should be withheld until hepatotoxicity is resolved, with subsequent stepwise rechallenge with the therapeutic drugs [11].

The dynamics of ADIH occurrence is unclear. Several studies have indicated that ADIH is most likely within the first month of the intensive phase of treatment [12- 
14]. Baseline alanine aminotransferase (ALT) measurement is recommended for patients suspected of liver disease, infection with human immunodeficiency virus, and pregnant or postpartum women [11]. ALT monitoring should be done between 2 and 4 weeks after initiation of treatment depending on the risk of hepatotoxicity and the stability of ALT. Baseline liver function tests are generally not recommended for healthy subjects. However, recent results indicated that universal testing should be done 2 weeks after treatment initiation in all patients during antituberculous therapy [15].

This study sought to clarify the necessity of intensive early monitoring for ADIH by investigating ADIH onset time and comparing the characteristics between early and late occurrence of ADIH.

\section{METHODS}

\section{Patients}

A total of 1,487 consecutive patients (> 18 years of age) who commenced antituberculous therapy at Gyeongsang National University Hospital between February 2009 to January 2013 were enrolled. Most patients received a 2-month treatment regimen of isoniazid, rifampicin, ethambutol, and pyrazinamide followed by isoniazid, rifampicin, and ethambutol for 4 months. All patients had received a fixed dose of first-line antituberculous drug daily according to World Health Organization recommendations based on weight [16]. Demographic and clinical data including age, sex, body mass index (BMI), concomitant diseases, infected organs with tuberculosis, previous history of tuberculosis, and alcohol consumption were retrospectively obtained from a review of medical records. Exclusion criteria were: (1) level of aspartate aminotransferase (AST) or ALT at baseline $>2$ times upper limit of normal (ULN; $\mathrm{n}=7$ ); (2) loss to follow-up within 2 months of treatment initiation $(\mathrm{n}=22 \mathrm{O})$; (3) inadequate date of laboratory testing at baseline or 2 to 4 weeks after treatment initiation $(\mathrm{n}=86)$; (4) diagnosis of latent tuberculosis, nontuberculous mycobacterium infection, multidrug resistant tuberculosis, or other disease ( $n=127$ ); and (5) discontinuation due to other adverse effects except hepatotoxicity $(n=16)$. Finally, 1,031 patients were recruited. The Institutional Review Board of Gyeongsang National University Hospital reviewed and approved this study.

\section{Definition of ADIH}

ADIH was defined as follows based on previous studies $[3,17]$. Other causes of elevated liver function tests were excluded. In cases with normal level of baseline AST and ALT before treatment initiation, liver transaminase levels were exceeded 3 times of upper normal range with symptoms of hepatitis or exceeded 5 times of upper normal range without symptoms. In cases with abnormal level of basal AST or ALT before treatment initiation, the level(s) after initiation of treatment was double the basal level. Level of total bilirubin was 2 times higher than ULN at the normal baseline or basal level at the abnormal baseline. Patients developing hepatotoxicity were categorized based on the timing of occurrence of $\mathrm{ADIH}$ ( $\leq$ 30-day group, ADIH detected within 30 days; > 30-day group, ADIH detected beyond 30 days). In subgroup analysis, patients developing hepatotoxicity were categorized according to occurrence within 7 days ( $\leq$ 7-day group) and beyond 7 days (> 7-day group).

\section{Monitoring and prognosis}

Liver function was ascertained at baseline and every 2 to 4 weeks after treatment initiation. Unplanned visits and laboratory tests due to adverse events were performed according to patient need. Elevated AST or ALT values prompted more frequent visits. In cases of $\mathrm{ADIH}$, all drugs were stopped and a step-wise rechallenge was begun after complete resolution of hepatotoxicity. During the presentation of hepatotoxicity, highest AST or ALT level and time of peak level were corrected. The prognosis of patients who successfully recommenced first-line drugs without further development of hepatitis, who began alternative regimens, or who terminated treatment were determined. Mean time to recovery was defined as the time interval between the onset of hepatitis and complete normalization.

\section{Statistical analyses}

All statistical analyses were performed with SPSS version 18.0 (SPSS Inc., Chicago, IL, USA). For between-group comparisons, the Mann-Whitney $U$ test was used for continuous variable, with the chi-square test or Fisher exact test (for situations with small frequencies) was used for categorical variables. Risk factors associated 
Table 1. Comparison of demographics, laboratory data, and comorbidities between patients with and without ADIH

\begin{tabular}{|c|c|c|c|c|}
\hline Variable & $\begin{array}{l}\text { Total patients } \\
\qquad(\mathrm{n}=1,031)\end{array}$ & $\begin{array}{l}\text { Without ADIH } \\
\qquad(n=923)\end{array}$ & $\begin{array}{l}\text { With ADIH } \\
\qquad(\mathrm{n}=108)\end{array}$ & $p$ value \\
\hline \multicolumn{5}{|l|}{ Demographic } \\
\hline Age, yr & $55 \cdot 5 \pm 18.3$ & $55 \cdot 3 \pm 18.2$ & $57.4 \pm 18.6$ & 0.298 \\
\hline Male sex & $608(59.0)$ & $546(59.2)$ & $62(57.4)$ & 0.727 \\
\hline Body mass index, kg/m² & $21.9 \pm 4.3$ & $21.9 \pm 4.1$ & $22.0 \pm 5.6$ & 0.641 \\
\hline \multicolumn{5}{|l|}{ Site of infection ${ }^{\mathrm{a}}$} \\
\hline Extrapulmonary TB & $319(30.9)$ & $279(30.2)$ & $40(37.0)$ & 0.147 \\
\hline Pulmonary TB & $743(72.1)$ & $665(72.0)$ & $78(72.2)$ & 0.969 \\
\hline \multicolumn{5}{|l|}{ Initial laboratory findings } \\
\hline Aspartate transaminase, U/L & $24 \cdot 3 \pm 10.6$ & $23.6 \pm 9.9$ & $30.2 \pm 14.2$ & $<0.001$ \\
\hline Alanine transaminase, U/L & $19.9 \pm 11.7$ & $19.4 \pm 11.3$ & $23.9 \pm 14.2$ & 0.001 \\
\hline Total bilirubin, mg/dL & $0.9 \pm 11.2$ & $0.9 \pm 11.8$ & $0.7 \pm 0.4$ & $<0.001$ \\
\hline \multicolumn{5}{|l|}{ Underlying disease } \\
\hline History of TB & $155(18.2)$ & $143(19.0)$ & $12(12.2)$ & 0.105 \\
\hline Hepatitis B virus & $50(5 \cdot 4)$ & $45(5 \cdot 5)$ & $5(4 \cdot 7)$ & 0.714 \\
\hline Hepatitis C virus & $27(2.9)$ & $16(2.0)$ & $11(10.3)$ & $<0.001$ \\
\hline Alcohol consumption & $119(14.4)$ & $102(13.9)$ & $17(17 \cdot 7)$ & 0.319 \\
\hline Diabetes mellitus & $174(20.3)$ & $157(20.8)$ & $17(17.0)$ & 0.379 \\
\hline End-stage renal disease & $22(3.0)$ & $21(3 \cdot 3)$ & $1(1.1)$ & 0.505 \\
\hline Malignancy & $122(16.4)$ & $103(15.8)$ & $19(20.9)$ & 0.218 \\
\hline
\end{tabular}

Values are presented as mean \pm SD or number $(\%)$.

ADIH, antituberculous drug-induced hepatotoxicity; TB, tuberculosis.

${ }^{a}$ Thirty-one patients were simultaneously diagnosed with pulmonary and extrapulmonary tuberculosis, and 15 patients had an unknown primary infection site.

with ADIH were determined by logistic regression analysis. The risk was expressed by calculating the odds ratio (OR) and 95\% confidential interval (CI). A p $<0.05$ indicated statistical significance.

\section{RESULTS}

\section{Baseline characteristics}

Mean age \pm standard deviation of the 1,031 patients $(608$ males, $59.0 \%$ ) was $55.5 \pm 18.3$ years (Table 1). One hundred and eight patients (10.5\%) developed ADIH a mean of $39.6 \pm 43.7$ days after treatment initiation. Baseline levels of AST and ALT in patients with ADIH were significantly higher than those in patients without ADIH. Baseline level of total bilirubin in patients with $\mathrm{ADIH}$ was significantly lower than that in patients without ADIH. Incidence of concomitant hepatitis $\mathrm{C}$ virus infection was
Table 2. Distribution of the infected organs with tuberculosis

\begin{tabular}{lc}
\hline Site of infection & No. $(\%)$ \\
\hline Lung & $710(68.9)$ \\
Pleura & $110(10.7)$ \\
Lymph node & $54(5.2)$ \\
Gastrointestinal tract or peritoneum & $42(4.1)$ \\
Central nervous system & $20(1.9)$ \\
Vertebral joint & $14(1.4)$ \\
Pericardium & $12(1.2)$ \\
Other site & $38(3.7)$ \\
Multiple site & $31(3.0)$ \\
\hline
\end{tabular}

significantly increased in patients with ADIH. However, the other variables were not significantly different. The most common infected site was the lung (68.9\%), followed by pleural space (10.7\%), lymph node (5.2\%), gas- 


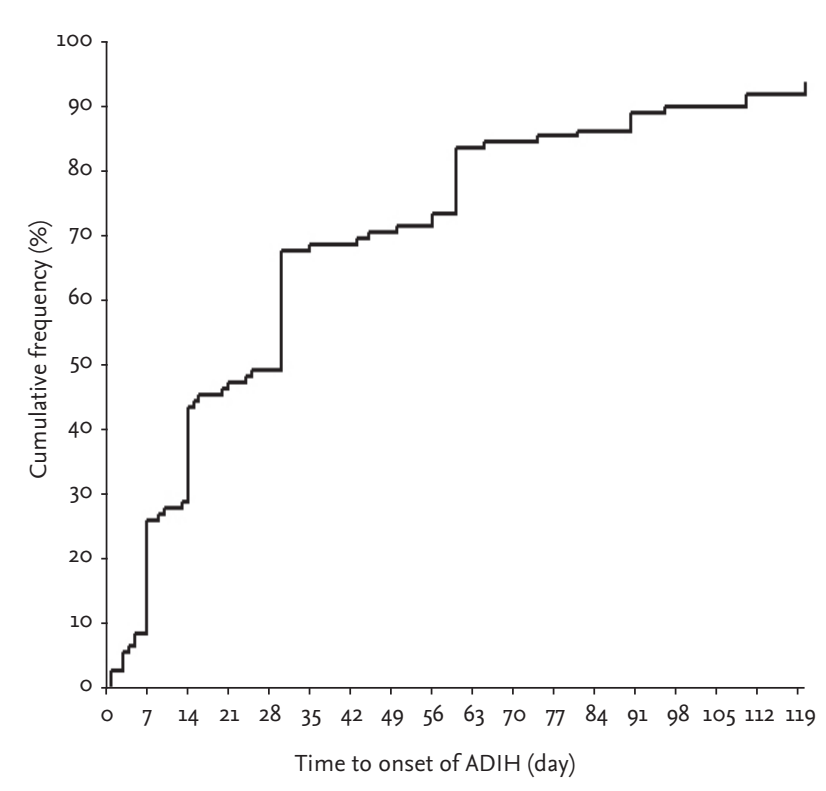

Figure 1. The time from the start of treatment to onset of hepatotoxicity in patients who developed antituberculous drug-induced hepatotoxicity (ADIH). Twenty-five percent and about $66 \%$ of the 108 patients developed ADIH within 7 and 30 days, respectively. Mean time to onset was 39.6 days (range, 1 to 200). trointestinal tract (4.1\%), central nervous system (1.9\%), vertebral joint (1.4\%), and pericardium (1.2\%) (Table 2 ). Thirty-one patients were simultaneously diagnosed with pulmonary and extrapulmonary tuberculosis.

\section{Onset time of ADIH}

Among 108 patients who developed ADIH, 73 patients (67.5\%) developed hepatotoxicity within 30 days (Fig. 1). The mean onset time of hepatotoxicity of the $\leq 30$-day group and > 30-day group was $15.6 \pm 10.1$ days and 89.7 \pm 44.4 days, respectively $(p<0.001)$. The mean peak AST, ALT, and bilirubin level was $301.4 \pm 350.0 \mathrm{U} / \mathrm{L}, 308.2 \pm$ $319.9 \mathrm{U} / \mathrm{L}$, and $1.5 \pm 1.7 \mathrm{mg} / \mathrm{mL}$, respectively, in the $\leq 30-$ day group and $241.5 \pm 393.5 \mathrm{U} / \mathrm{L}, 211.5 \pm 230.1 \mathrm{U} / \mathrm{L}$, and $2.4 \pm 3.4 \mathrm{mg} / \mathrm{mL}$, respectively, in the > 30-day group. Except for the peak ALT level, there were no significant differences in the other variables between the two groups (Table 3).

Twenty-eight patients (25.9\%) developed hepatotoxicity within 7 days. The mean hepatitis onset time of the $\leq$ 7-day group and $>7$-day group was $5.7 \pm 2.1$ days and 51.4 \pm 45.1 days, respectively $(p<0.001)$. In the $\leq 7$-day group, rates of extrapulmonary tuberculosis were significantly

Table 3. Clinical and laboratory characteristics of patients developing antituberculous drug-induced hepatotoxicity in $\leq 30-$ day group and $>30$-day group

\begin{tabular}{|c|c|c|c|}
\hline Variable & $\leq 30$-Day group $(n=73)$ & $>30$-Day group $(\mathrm{n}=35)$ & p value \\
\hline \multicolumn{4}{|l|}{ Site of infection ${ }^{\mathrm{a}}$} \\
\hline Extrapulmonary TB & $29(39.7)$ & $11(31.4)$ & 0.403 \\
\hline Pulmonary TB & $53(72.6)$ & $25(71.4)$ & 0.899 \\
\hline \multicolumn{4}{|l|}{ Initial laboratory finding } \\
\hline AST, U/L & $30.7 \pm 13.6$ & $29.2 \pm 15.7$ & 0.267 \\
\hline ALT, U/L & $24.8 \pm 14.6$ & $22.0 \pm 13.3$ & 0.331 \\
\hline Total bilirubin, mg/dL & $0.7 \pm 0.4$ & $0.7 \pm 0.4$ & 0.468 \\
\hline \multicolumn{4}{|l|}{ Laboratory finding during treatment } \\
\hline Peak AST, U/L & $301.4 \pm 350.0$ & $241.5 \pm 393.5$ & 0.177 \\
\hline Peak ALT, U/L & $308.2 \pm 319.9$ & $211.5 \pm 230.1$ & 0.038 \\
\hline Peak total bilirubin, $\mathrm{mg} / \mathrm{dL}$ & $1.5 \pm 1.7$ & $2.4 \pm 3.4$ & 0.233 \\
\hline Modes of management & & & 0.033 \\
\hline Maintenance of 1st line drugs & $50(68.1)$ & $20(57.1)$ & \\
\hline Change of regimen or termination of treatment & $23(31.9)$ & $15(42.9)$ & \\
\hline Time to recovery, day & $40.8 \pm 34.4$ & $47 \cdot 4 \pm 49 \cdot 3$ & 0.755 \\
\hline
\end{tabular}

Values are presented as number (\%) or mean \pm SD.

TB, tuberculosis; AST, aspartate transaminase; ALT, alanine transaminase.

${ }^{a}$ Ten patients were simultaneously diagnosed with pulmonary and extrapulmonary tuberculosis. 
Table 4. Subgroup analysis of patients developing antituberculous drug-induced hepatotoxicity between the $\leq 7$-day group and > 7-day group

\begin{tabular}{|c|c|c|c|}
\hline Variable & $\leq 7$-Day group $(\mathrm{n}=28)$ & > 7-Day group $(\mathrm{n}=80)$ & $p$ value \\
\hline \multicolumn{4}{|l|}{ Site of infection ${ }^{a}$} \\
\hline Extrapulmonary TB & $15(53 \cdot 6)$ & $25(31.3)$ & 0.035 \\
\hline Pulmonary TB & $15(53 \cdot 6)$ & $63(78.8)$ & 0.010 \\
\hline \multicolumn{4}{|l|}{ Initial laboratory findings } \\
\hline AST, U/L & $34.1 \pm 14.4$ & $28.9 \pm 14.0$ & 0.036 \\
\hline $\mathrm{ALT}, \mathrm{U} / \mathrm{L}$ & $30.1 \pm 17.2$ & $21.7 \pm 12.4$ & 0.013 \\
\hline Total bilirubin, mg/dL & $0.7 \pm 0.4$ & $0.7 \pm 0.4$ & 0.594 \\
\hline \multicolumn{4}{|l|}{ Laboratory findings during treatment } \\
\hline Peak AST, U/L & $183.9 \pm 149.9$ & $316.3 \pm 408.7$ & 0.064 \\
\hline Peak ALT, U/L & $188.5 \pm 130.8$ & $307.8 \pm 330.6$ & 0.129 \\
\hline Peak total bilirubin, mg/dL & $1.5 \pm 1.7$ & $2.0 \pm 2.7$ & 0.939 \\
\hline Modes of management & & & 0.033 \\
\hline Maintenance of 1st line drugs & $23(81.5)$ & $47(58.7)$ & \\
\hline Change of regimen or termination of treatment & $5(18.5)$ & $33(41.3)$ & \\
\hline Time to recovery, day & $29 \cdot 3 \pm 25.2$ & $47.5 \pm 41.9$ & 0.209 \\
\hline
\end{tabular}

Values are presented as number (\%) or mean \pm SD.

TB, tuberculosis; AST, aspartate transaminase; ALT, alanine transaminase.

${ }^{a}$ Ten patients simultaneously were diagnosed with pulmonary and extrapulmonary tuberculosis.

higher than those of the > 7-day group (53.6\% vs. $31.3 \%$, $p=0.035$ ) (Table 4). Among the 40 patients with extrapulmonary tuberculosis, 15 patients (37.5\%) developed hepatotoxicity within 7 days. There were no significant differences of peak AST and ALT levels between the $\leq$ 7- and > 7-day groups. But, the mean AST and ALT levels at baseline were significantly higher in the $\leq 7$-day group compared to the > 7-day group (34.1 \pm 14.4 vs. $28.9 \pm 14.0$, $p=0.036 ; 30.1 \pm 17.2$ vs. $21.7 \pm 12.4, p=0.013$, respectively) (Table 3). However, there were no significant differences in age, sex, BMI, pyrazinamide (PZA) combination, total bilirubin level at baseline and peak time, concomitant hepatitis B virus and hepatitis C virus infection, alcohol consumption, diabetes, and treatment strategy between the two groups.

\section{Clinical course and management}

As shown in Table 5 serum ALT > 40 IU/L (OR, 4.442; 95\% CI, 2.594 to $7.604 ; p<0.001$ ), serum AST > $40 \mathrm{IU} / \mathrm{L}$ (OR, 2.609; 95\% CI, 1.361 to 5.003; $p=0.004$ ), serum bilirubin > $1.2 \mathrm{mg} / \mathrm{dL}$ (OR, 2.270; 95\% CI, 1.060 to 4.861 ), and presence of anti-hepatitis C virus (OR, 5.701; 95\% CI, 2.571 to $12.640 ; p<0.001$ ) were significantly associated with development of ADIH in univariate analysis. In

Table 5. Logistic regression analysis of risk factors for developing antituberculous drug-induced hepatotoxicity

\begin{tabular}{|c|c|c|c|c|}
\hline \multirow{2}{*}{ Variable } & \multicolumn{2}{|c|}{ Univariate analysis } & \multicolumn{2}{|c|}{ Multivariate analysis } \\
\hline & OR $(95 \% \mathrm{CI})$ & $p$ value & OR $(95 \% \mathrm{CI})$ & $p$ value \\
\hline $\mathrm{ALT}>40 \mathrm{IU} / \mathrm{L}$ & $4.442(2.594-7.604)$ & $<0.001$ & $2.995(1.580-5.680)$ & 0.001 \\
\hline $\mathrm{AST}>40 \mathrm{IU} / \mathrm{L}$ & $2.609(1.361-5.003)$ & 0.004 & $1.213(0.556-2.648)$ & 0.628 \\
\hline Bilirubin > $1.2 \mathrm{mg} / \mathrm{dL}$ & $2.270(1.060-4.861)$ & 0.035 & $1.550(0.690-3.482)$ & 0.288 \\
\hline Anti-hepatitis C virus & $5.701(2.571-12.640)$ & $<0.001$ & $4.204(1.822-9.700)$ & 0.001 \\
\hline
\end{tabular}

OR, odds ratio, CI, confidential interval; ALT, alanine transaminase; AST, aspartate transaminase. 
multivariate analysis, serum ALT > $40 \mathrm{IU} / \mathrm{L}$ (OR, 2.995; 95\% CI, 1.580 to $5.680 ; p=0.001)$ and presence of anti-hepatitis C virus (OR, 4.204; 95\% CI, 1.822 to 9.700; $p=$ 0.001 ) were independent risk factors for development of $\mathrm{ADIH}$. There was no significant difference in time to recovery between the $\leq 30$ - and the $>30$-day groups $(40.8 \pm$ 34.4 and $47.4 \pm 49.3$ days, $p=0.755)$. In the $\leq 30$-day group, 50 patients $(68.1 \%)$ successfully completed treatment with first-line drugs without further hepatotoxicity development, and 23 patients (31.9\%) commenced treatment with alternative drugs or terminated treatment. In the > 30-day group, 20 patients $(57.1 \%)$ successfully completed treatment with first-line drugs, and 15 patients (42.9\%) commenced alternative drug treatment or terminated treatment (Table 3). There was a significant difference in modes of management in both groups $(p=$ 0.033). In the $\leq 7$-day group, the proportion of patients who maintained first-line antituberculous drugs without withdrawal or termination was higher than that with patients in whom ADIH occurred later $(81.5 \%$ vs. $58.7 \%$, $p=0.033$ ) (Table 4) and ADIH occurred between 8 and 14 days $(81.5 \%$ vs. $18.5 \%, p=0.036$, data not shown). There were no cases of acute liver failure requiring liver transplantation or death.

\section{DISCUSSION}

In current study, hepatotoxicity occurred in $10.5 \%$ of patients who received antituberculous therapy. In these patients, the mean onset time of ADIH was 39.6 days after treatment initiation. The majority of ADIH events appeared within the first 30 days of treatment. In these patients, the peak ALT level was higher and there were more patients who maintained first-line antituberculous drugs compared to patients who developed $\mathrm{ADIH}$ more than 30 days after initiation of treatment. Patients in whom ADIH develop within 7 days of treatment initiation comprised more patients who maintained first-line antituberculous drug therapy and a higher proportion of patients with extrapulmonary tuberculosis compared to patients in whom ADIH develop more than 7 days after treatment began. Therefore, early identification of $\mathrm{ADIH}$ is clinically important for maintenance of first-line antituberculous treatment without a change of regimen. Emphasizing this importance, 37.5\% of patients with extrapulmonary tuberculosis developed hepatotoxicity within 7 days.

Early detection of ADIH is clinically important for several reasons. Earlier detection of ADIH has been associated with decreased mortality and severe hepatotoxicity [18,19]. Moreover, earlier detection of ADIH may allow a shorter time to recovery, from occurrence of ADIH to normalization of AST, ALT, and bilirubin, because resolution of liver function test abnormality is likely to be faster. In a recent study, a uniform monitoring policy at 2 weeks in all patients treated for active tuberculosis was useful for prompt detection of a subgroup of patients who developed ADIH early [15]. Presently, earlier detection of hepatotoxicity enabled patients with antituberculous treatment to maintain the first-line drug regimen compared to later detection. However, regardless of the cutoff date ( 7 or 30 days), there was no significant difference in time to recovery between earlier versus later detection.

ADIH occurred in $10.5 \%$, similar to previously reported rates of $3 \%$ in Canada [17], $5 \%$ in Hong Kong [20], 5.3\% in Singapore [21], 16.1\% in Taiwan [22], 2.6\% in China [7], and $10.5 \%$ in India [18]. The present mean onset time of ADIH was 39.6 days, which is similar to reported median onset times of 14 days in Turkey [23], 38 days in Singapore [21], and 53 days in China [7]. A cohort study conducted in India showed that $35 \%$ of the cases of ADIH developed within 15 days and 58\% within 30 days [18]. In another prospective study, $58 \%$ of 74 patients with ADIH developed the hepatotoxicity within the first 2 weeks [13], similar to the present findings. The Indian study reported that ADIH in 69 patients progressed to acute liver failure in 11 patients (16\%) within 7 days and in $18(26.1 \%)$ within 14 days. However, there were no cases of acute liver failure requiring liver transplantation or death in our study.

As reported in previous studies $[8,15,24,25]$, poor liver function at baseline and hepatitis $\mathrm{C}$ virus infection were independent risk factors for development of ADIH (Table 5). However, there was no significant difference between the $\leq 30$ - and $>30$-day groups in baseline liver function and hepatitis $\mathrm{C}$ virus infection. However, the mean AST and ALT levels at baseline were higher significantly in the $\leq 7$-day group than in the $>7$-day group.

In addition, a high proportion of patients (37.5\%) with extrapulmonary tuberculosis developed hepatotoxicity 
within 7 days. Among the 319 patients with extrapulmonary tuberculosis, 40 patients (12.7\%) developed ADIH and $37.0 \%$ of these cases (15/40) occurred within 7 days. These findings suggest that monitoring liver function during the first week in patients with extrapulmonary tuberculosis and abnormal liver function at baseline could be helpful for very early detection of ADIH and improved prognosis.

Our study had several limitations. It was a retrospective study. Precise information on alcohol consumption and hepatotoxic drugs were unavailable from a medical chart review. We did not measure hepatotoxicity in all patients during the first 7 days, and proportion of $\mathrm{ADIH}$ in this group may be underestimated. Secondly, we did not investigate 456 excluded patients, which could create an exclusion bias. Third, because the study was undertaken at the national referral center, our results may not be generalizable.

In conclusion, it is important to start monitoring liver function test earlier in patients undergoing antituberculous treatment, because early identification of ADIH is associated with completion of treatment using firstline antituberculous drugs. Our results suggest that clinicians should consider liver function testing during the first 7 days of treatment in patients with extrapulmonary tuberculosis or poor baseline liver function, since this may improve prognosis. Meticulous attention should be given to the patients with high ALT and/or anti-hepatitis $\mathrm{C}$ virus.

\section{KEY MESSAGE}

1. Of the 1,031 patients, hepatotoxicity occurred in $10.5 \%$ of patients who received antituberculous therapy.

2. The majority (67.6\%) of hepatotoxicity events appeared within the first 30 days of treatment.

3. Earlier detection of hepatotoxicity enabled patients with antituberculous treatment to maintain the first-line drug regimen compared to later detection.

\section{Conflict of interest}

No potential conflict of interest relevant to this article was reported.

\section{REFERENCES}

1. World Health Organization. Global tuberculosis report 2013 [Internet]. Geneva: World Health Organizaton, 2013 [cited 2015 Sep 10]. Available from: http://apps.who.int/ iris/bitstream/10665/91355/1/9789241564656_eng.pdf.

2. National Collaborating Centre for Chronic Conditions (UK); Centre for Clinical Practice at NICE (UK). Tuberculosis: clinical diagnosis and management of tuberculosis, and measures for its prevention and control. London: National Institute for Health and Clinical Excellence (NICE), 2011.

3. Hovell MF, Sipan CL, Blumberg EJ, et al. Increasing Latino adolescents' adherence to treatment for latent tuberculosis infection: a controlled trial. Am J Public Health 2003;93:1871-1877.

4. Gangadharam PR. Isoniazid, rifampin, and hepatotoxicity. Am Rev Respir Dis 1986;133:963-965.

5. Schaberg T, Rebhan K, Lode H. Risk factors for side-effects of isoniazid, rifampin and pyrazinamide in patients hospitalized for pulmonary tuberculosis. Eur Respir J 1996;9:2026-2030.

6. Yew WW, Leung CC. Antituberculosis drugs and hepatotoxicity. Respirology 2006;11:699-707.

7. Shang P, Xia Y, Liu F, et al. Incidence, clinical features and impact on anti-tuberculosis treatment of anti-tuberculosis drug induced liver injury (ATLI) in China. PLoS One 2011;6:e21836.

8. Sun HY, Chen YJ, Gau CS, Chang SC, Luh KT. A prospective study of hepatitis during antituberculous treatment in Taiwanese patients and a review of the literature. J Formos Med Assoc 2009;108:102-111.

9. Makhlouf HA, Helmy A, Fawzy E, El-Attar M, Rashed HA. A prospective study of antituberculous drug-induced hepatotoxicity in an area endemic for liver diseases. Hepatol Int 2008;2:353-360.

10. Lorent N, Sebatunzi O, Mukeshimana G, Van den Ende J, Clerinx J. Incidence and risk factors of serious adverse events during antituberculous treatment in Rwanda: a prospective cohort study. PLoS One 2011;6:e19566.

11. Saukkonen JJ, Cohn DL, Jasmer RM, et al. An official ATS statement: hepatotoxicity of antituberculosis therapy. Am J Respir Crit Care Med 2006;174:935-952.

12. Ormerod LP, Skinner C, Wales J. Hepatotoxicity of antituberculosis drugs. Thorax 1996;51:111-113.

13. Senaratne WV, Pinidiyapathirage MJ, Perera GA, Wick- 
remasinghe AR. Anti-tuberculosis drug inducd hepatitis a Sri Lankan experience. Ceylon Med J 2006;51:9-14.

14. Baghaei P, Tabarsi P, Chitsaz E, et al. Incidence, clinical and epidemiological risk factors, and outcome of drug-induced hepatitis due to antituberculous agents in new tuberculosis cases. Am J Ther 2010;17:17-22.

15. Singanayagam A, Sridhar S, Dhariwal J, et al. A comparison between two strategies for monitoring hepatic function during antituberculous therapy. Am J Respir Crit Care Med 2012;185:653-659.

16. World Health Organization. Treatment of Tuberculosis: Guidelines. 4th ed. Geneva: World Health Organization, 2010.

17. Yee D, Valiquette C, Pelletier M, Parisien I, Rocher I, Menzies D. Incidence of serious side effects from first-line antituberculosis drugs among patients treated for active tuberculosis. Am J Respir Crit Care Med 2003;167:14721477 .

18. Agal S, Baijal R, Pramanik S, et al. Monitoring and management of antituberculosis drug induced hepatotoxicity. J Gastroenterol Hepatol 2005;20:1745-1752.

19. McNeill L, Allen M, Estrada C, Cook P. Pyrazinamide and rifampin vs isoniazid for the treatment of latent tubercu- losis: improved completion rates but more hepatotoxicity. Chest 2003;123:102-106.

20. Chang KC, Leung CC, Yew WW, Lau TY, Tam CM. Hepatotoxicity of pyrazinamide: cohort and case-control analyses. Am J Respir Crit Care Med 2008;177:1391-1396.

21. Teleman MD, Chee CB, Earnest A, Wang YT. Hepatotoxicity of tuberculosis chemotherapy under general programme conditions in Singapore. Int J Tuberc Lung Dis 2002;6:699-705.

22. Huang YS, Chern HD, Su WJ, et al. Cytochrome P450 2E1 genotype and the susceptibility to antituberculosis drug-induced hepatitis. Hepatology 2003;37:924-930.

23. Gulbay BE, Gurkan OU, Yildiz OA, et al. Side effects due to primary antituberculosis drugs during the initial phase of therapy in 1149 hospitalized patients for tuberculosis. Respir Med 2006;100:1834-1842.

24. Lomtadze N, Kupreishvili L, Salakaia A, et al. Hepatitis C virus co-infection increases the risk of anti-tuberculosis drug-induced hepatotoxicity among patients with pulmonary tuberculosis. PLoS One 2013;8:e83892.

25. Liu YM, Cheng YJ, Li YL, Liu CE, Hsu WH. Antituberculosis treatment and hepatotoxicity in patients with chronic viral hepatitis. Lung 2014;192:205-210. 